\title{
Photometry of Galaxies on Kiso Schmidt Plates
}

\author{
Takashi Ichikawa \\ Kiso Observatory, The University of Tokyo, Mitake-mura, Kiso-gun, \\ Nagano 397-01, Japan
}

\begin{abstract}
In spite of the lower $\mathrm{S} / \mathrm{N}$ and smaller dynamic range of photographic plates compared to those of CCD data, Schmidt plates still have the advantage of a large field of view, which allows us to study the structure of nearby large galaxies and clusters of galaxies. Investigating the internal and external errors of our surface photometry, we show that a careful analysis of photographic plates results in reliable surface photometry data comparable to CCD data for galaxies brighter than $\sim 17 \mathrm{mag}$. Recent studies based on photographic surface photometry catalogues obtained from Kiso Schmidt plates are reviewed and it can be shown that surface photometry of galaxies on Schmidt plates is still useful for understanding the local universe.
\end{abstract}

\section{Introduction}

Studies of the physical structure and the distribution of nearby galaxies and clusters of galaxies are important for understanding the local universe. Recent comprehensive investigations on the general nature of field galaxies and clusters of galaxies, cosmic distance scales, peculiar velocity fields, and kinematics of cluster of galaxies inevitably involve statistical analyses of a number of galaxies requiring accurate and homogeneous observational data and photometric parameters derived in a consistent system. In the late 1970s, Kodaira and his colleagues started a project to obtain homogeneous surface photometric data for as many nearby bright galaxies as possible, using the $105 \mathrm{~cm}$ Schmidt telescope at Kiso Observatory. Initial targets of the project were members of the Virgo and Ursa Major clusters of galaxies. Surface photometry data of 261 galaxies in the $V$ band were first compiled by Watanabe (1983). Since then, we have focused mainly on nearby galaxies brighter than $\sim 17$ mag.

Beginning in 1984, the above mentioned project was extended to cover most of the northern bright galaxies whose structure can be reasonably resolved on Kiso Schmidt plates. Efforts were made to secure a homogeneous database which can be used for studies of the Local Supercluster. The target galaxies were selected from the RSA catalogue (Sandage \& Tammann 1981). This effort has resulted in The Photometric Atlas of Northern Bright Galaxies (Kodaira et al. 1990, hereafter PANBG). The PANBG database contains photometric parameters as well as images, isophotes, and profiles of 791 galaxies in 256 areas, and it is one of the largest homogeneous surface photometry databases of northern bright galaxies. 
Another complete catalogue of the Virgo cluster was prepared by Yasuda et al. (1994) in the $B$ band. The catalogue contains virtually all spirals (246 galaxies in 8 areas) ever used in published studies of the Tully-Fisher relation. Recently, Ichikawa \& Watanabe (1994) obtained $B$-band surface photometry of $\sim 1900$ galaxies in the $\sim 900$ square degree Pisces-Perseus region at $22^{h}<\alpha<$ $4^{h}$ and $22^{\circ}<\delta<33^{\circ}$. The catalogue includes all CGCG, UGC, and optically identified IRAS galaxies (hereafter, P-P catalogue). The observations have been extended to the CfA region $\left(8^{h}<\alpha<17^{h}\right.$ and $\left.25^{\circ}<\delta<37^{\circ}\right)$, for which the analysis is in progress.

It is sometimes claimed that surface photometry using photographic plates is out of date, and less reliable than that obtained with CCD cameras. It is noteworthy, however, that a careful analysis leads to reliable datasets with an accuracy comparable to that of CCD data, at least for bright nearby galaxies. Moreover, Schmidt plates still have the advantage of their large field of view. Here we will describe the surface photometry obtained with Kiso Schmidt plates, following Kodaira et al. (1990), Ichikawa \& Fukugita (1992), and Ichikawa \& Watanabe (1994). The internal and external accuracy is described in detail and some scientific results derived from these catalogues are reviewed as well. We will focus on surface photometry based on measurements of individually selected galaxies and we will therefore not go into the details of studies based on automatic detections using full-plate scanning (e.g., Yamagata 1986; Doi 1992)

\section{Observations}

The observations discussed were obtained with the $105 \mathrm{~cm}$ Kiso Schmidt telescope, which has a focal length of $3300 \mathrm{~mm}$, giving an image scale of $62.5^{\prime \prime} \mathrm{mm}^{-1}$. A 6-degree square field is covered by a 14-inch photographic plate. Mainly the $B$ and $V$ bands were used for wide area observations, since the sky background limit is reached faster than in the $R$ and $I$ bands. Standard exposure times are 20 to 50 minutes, but the actual exposure time depends strongly upon the sky brightness, plate sensitivity, and hypersensitization. Kodak IIa-D or 103aD emulsions are combined with a Schott GG495 glass filter to define the photographic $V$ band, and the $B$ band is defined with $\mathrm{IIaO}$ or $103 \mathrm{aO}$ emulsions and a GG385 filter. The standard celestial area system used for the Kiso Schmidt has slight overlaps between adjacent areas. Therefore galaxies in adjacent areas were photographed on more than two plates and can be used for checking the internal accuracy of the surface photometry. After an area is photographed, the four sets of step wedges, each consisting of 15 steps, are exposed on the margin of the plate for calibration purposes.

\section{Plate Measurement}

The scanning area of each target galaxy identified on the plates is defined according to its apparent size. For each galaxy, a sufficiently wide area was scanned to allow for an accurate determination of the sky background. Galaxy images were scanned with a PDS 2020GMS microdensitometer at Kiso Observatory. A logarithmic amplifier was devised for the PDS 2020GMS, which is five times faster than the Perkin-Elmer amplifier originally built for the PDS (Anderson 
et al. 1983). An efficient PDS control program was developed, which allows us to scan many galaxies continuously on a Schmidt plate. The output of a galaxy scan is a digital array of photographic densities. The step wedges of each plate were scanned before and after galaxy scans for monitoring the possible drift in the response of the PDS.

\section{Data reduction}

The data reduction is carried out using the SPIRAL software library developed at Kiso Observatory (Ichikawa et al. 1987; Hamabe and Ichikawa 1992) in an IRAF environment. Special care is taken of density saturation in galactic centers, sky subtraction, cleaning, absolute calibration, and template fitting.

(1) Density saturation: Density saturation due to the small dynamic range is one of the most serious limitations of photographic plates, and it is sometimes prone to limit reliable photometry. (A small dynamic range of the PDS may be partly related to the limitations as well.) The average seeing size at Kiso Observatory is about $3.5^{\prime \prime}$, which tends to smear the central peak of galaxies. Although the large seeing size hides the fine structure of galaxies, it prevents the central saturation. In fact, saturated galaxies are rare.

(2) Sky subtraction: The pixel values in a density frame are converted, pixel by pixel, to intensities, using the characteristic curve of a plate to produce an intensity frame. The sky background is determined by fitting a two-dimensional polynomial in the regions not covered by the galaxy, bright stars, and neighbouring galaxies. Sky subtractions are made using zeroth (constant subtraction) to fifth order polynomials. The most appropriate order of the polynomial is selected visually. A first order polynomial, i.e., an inclined plane, is usually applied for small galaxies. Large galaxies sometimes require higher order polynomials. Then the sky background, approximated by the polynomial of the adopted order, is subtracted, pixel by pixel, from the galaxy frame. The resultant galaxy frame is divided, again pixel by pixel, by the sky background to yield a relative intensity frame of the galaxy. In this process the vignetting is corrected by assuming constant sky background over the plate.

(3) Absolute calibration: The unit of relative intensity, i.e., the sky brightness, can be determined with aperture photometry data (e.g., Longo and de Vaucouleurs 1983) or CCD data. Photoelectric magnitudes of a galaxy measured with various apertures are compared with the photographic magnitudes integrated within the same apertures. In practice, the run of photographic magnitudes as a function of the aperture size, i.e., the growth curve, is fitted by the least squares method to the photoelectric magnitudes.

The mean sky brightness for a plate is computed as a weighted mean of the sky brightnesses determined for the calibrators on a plate. The implicit assumption for this procedure is that the actual sky brightness is constant over the 6-degree field covered by a Schmidt plate.

(4) Cleaning: Field stars are removed from galaxy frames automatically or interactively to produce cleaned frames. Neighbouring galaxies, if any, plate faults, 
scratches, and trails of artificial satellites seen occasionally in a frame are also cleaned out interactively. The values of the pixels inside stellar images or in any unwanted images are replaced with the values interpolated from the surrounding pixels. In the case where a galaxy overlaps with an objective galaxy, the galaxies were decomposed interactively, keeping the sum of the luminosities unchanged (see PANBG). Some galaxies are deeply embedded in envelopes of neighbouring bright galaxies, or in photographic halos of bright stars. Photometric parameters of such galaxies are inevitably subject to large uncertainties.

(5) Total magnitude and effective radius: After subtracting the sky background and cleaning stellar images and plates defects, we measured the intensity of a galaxy on circular annuli outward from the galaxy center with one pixel interval, thus obtaining a growth curve for each galaxy. The morphology-dependent template curves given by de Vaucouleurs (1977) or the RC3 were adjusted to the growth curves, in order to derive the total magnitude and the effective radius as defined in the RC2.

Since template growth curves for the $V$ band were not available, Kodaira et al. (1990) constructed them by averaging the growth curves of nearby galaxies, divided into two inclination classes. For each inclination class the average growth curves were obtained for six morphological types to be used in measuring $V$-band total magnitudes.

(6) Surface photometric parameters: Cleaned frames are subjected to surface photometric analyses. An ellipse fitting program similar to that described by Lauer (1985) was developed to derive surface photometric parameters. Isophotal parameters were derived by fitting an ellipse to the isophotes of certain levels (e.g., $25 \mathrm{mag} \operatorname{arcsec}^{-2}$ ) with the center of the ellipse left free in the fitting. The derived parameters are an integrated magnitude within the ellipse, semi-major and semi-minor axes, an axis ratio, the position angle of the major axis, and several other parameters. Contour maps and surface brightness profiles are also given in PANBG (Fig. 1).

\section{Photometric Accuracy}

(1) Internal consistency: The person-to-person consistency was checked through analyses made by different persons. In the P-P catalogue ( $B$ band) the consistency in the total magnitude was a $0.03 \mathrm{mag}$ offset and a $0.06 \mathrm{mag}$ dispersion for 157 galaxies. In PANBG ( $V$ band) the dispersions are $0.07 \mathrm{mag}$ in the total magnitude and 0.022 in the logarithm of the effective radius. The errors are likely to be due to the interactive analysis, which needs certain personal judgments such as sky subtraction, cleaning, and template fitting. This kind of error may be inevitable in the analysis of CCD data as well.

Galaxies photographed on two or more plates were used to estimate the internal consistency. These independent observations were subjected to independent data reduction. The standard deviation in total magnitudes was 0.10 mag in PANBG and $0.09 \mathrm{mag}$ in the P-P catalogue. For quite a few galaxies, photoelectric magnitudes were found to be poorly determined. The standard 

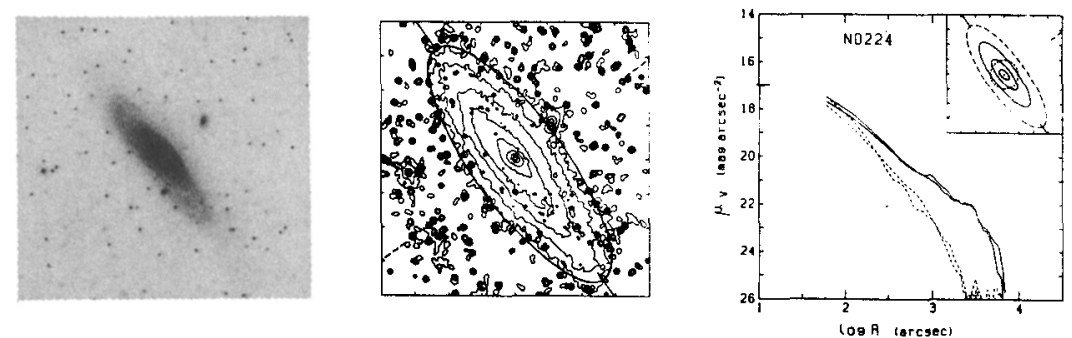

Figure 1. The data on M31 in the PANBG (Kodaira et al. 1990): intensity image (left), contour map (middle), and radial profiles (right).

deviation from the growth curves is larger than $0.1 \mathrm{mag}$. We recognize their error in the absolute calibration $( \pm 0.10-0.15 \mathrm{mag})$ is the major source of the error in the total magnitude. This fact will be confirmed by more accurate CCD photometry.

We made CCD observations of about 50 galaxies in 14 areas in the P-P region along with standard stars during photometric nights (Ichikawa \& Watanabe 1994). After adjusting for the difference in seeing size, the growth curves of photographic images were found to be in good agreement with those derived from CCD images. The scatter of the sky brightness obtained from CCD data was $\sim 0.06 \mathrm{mag}$ on average. This fact suggests that CCD photometry provides a more accurate calibration than photoelectric aperture photometry data.

(2) External errors: There are few catalogues based on homogeneous photometry which can be used to evaluate the external accuracy of our photometry. First we used the RC3 which contains the largest photometric data set compiled from the literature, transformed statistically to a homogeneous system. In a sample of Virgo cluster galaxies, Yasuda et al. (1994) found that $B_{\mathrm{T}}(\mathrm{RC} 3)=$ $B_{\mathrm{T}}$ (Kiso) $-0.08 \mathrm{mag}$ with a dispersion $\sigma=0.12$ (Fig. 2a). In the P-P region, the consistency is a $0.06 \mathrm{mag}$ offset and a $0.017 \mathrm{mag}$ dispersion (Fig. 2b).

We found that our photometric data are in good agreement with the RC3 for the galaxies brighter than $\sim 17 \mathrm{mag}$. Considering that the RC3 data are likely to have errors as large as ours, a comparison with more accurate photometry data is important. A few CCD observations are available in the catalogues of Cornell et al. (1987) and Pierce and Tully $\left(1988\right.$, PT) for $m_{B}<16$ mag. PT's catalogue is one of the most reliable homogeneous catalogues made with CCD observations. A comparison of the Virgo data by Yasuda et al. (1994) with CCD photometry data for 33 galaxies gives a regression line of $B_{\mathrm{T}}(\mathrm{PT})=B_{\mathrm{T}}$ (Kiso) $-0.01 \mathrm{mag}$ with 

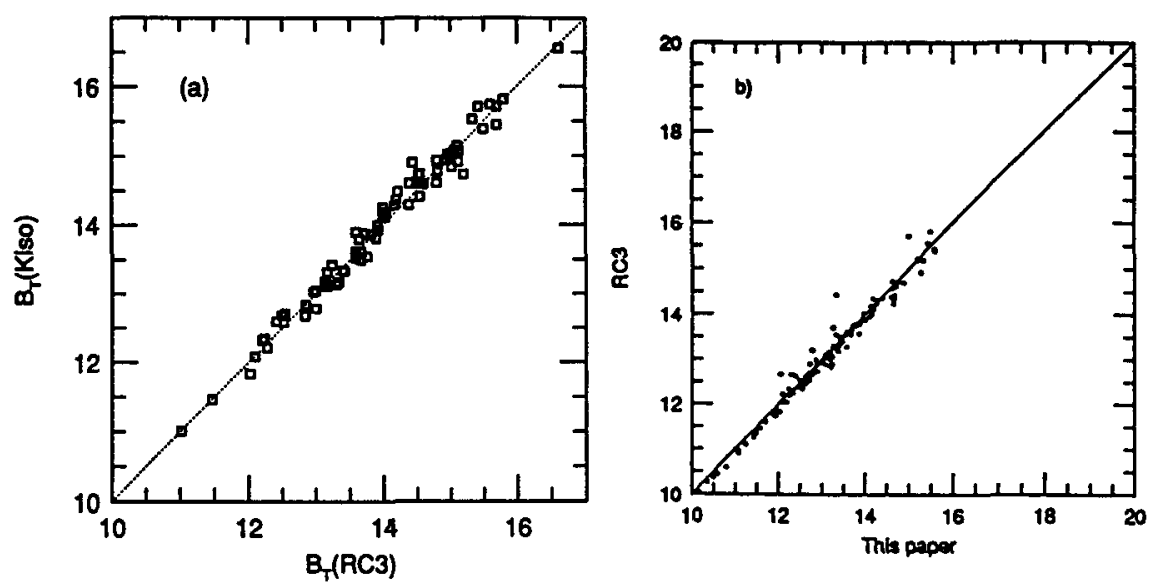

Figure 2. A Comparison of total magnitudes from the RC3 with those obtained from Kiso Schmidt plates: Virgo cluster galaxies by Yasuda et al. (1994) (right) and the Pisces-Perseus region by Ichikawa $\&$ Watanabe (1994) (left)

a dispersion $\sigma=0.12$. The consistency is better than that of the RC3, indicating that our photographic photometry yields magnitudes with an accuracy of $\sim 0.1$ mag with a quite small offset, at least for nearby galaxies $\left(m_{B}<16 \mathrm{mag}\right)$.

We also confirmed a good agreement between the axial ratios from Kiso photometry, the RC3, and PT. In terms of inclination we find $i(\mathrm{RC} 3)=i$ (Kiso) $-0.1^{\circ}\left(\sigma=7.2^{\circ}\right)$ and $i(\mathrm{PT})=i($ Kiso $)-1.1^{\circ}\left(\sigma=3.9^{\circ}\right)$ (Yasuda et al. 1994).

Cornell et al. (1987) gave CCD photometry of 9 galaxies in the P-P region. Their isophotal magnitudes, sizes, and axial ratios were also compared to our data. The isophotal magnitudes of two galaxies are inconsistent with our data, which we believe to be due to the CCD data, since our data are in rather good agreement with the RC3 data. Excluding these two galaxies, the isophotal magnitudes at $25 \mathrm{mag} \operatorname{arcsec}^{-2}$ of Cornell et al. (1987) have an average offset of $0.05 \mathrm{mag}$ from our results, but the dispersion is very small $(0.04 \mathrm{mag})$. It is therefore concluded that from surface photometry using Kiso Schmidt plates homogeneous catalogues can be produced with an accuracy of $0.1 \mathrm{mag}$ in total magnitude for nearby galaxies.

\section{Recent Scientific Results}

The intrinsic dispersion of the Tully-Fisher relation is about $0.35-0.40 \mathrm{mag}$ in the $B$ band, which is much larger than the photometric error of the Kiso Schmidt plates. Therefore, our data are accurate enough for application in a Tully-Fisher analysis. Combining photometry data in the P-P catalogue with redshift and $21 \mathrm{~cm}$ HI linewidth data for spiral galaxies at $22^{h}<\alpha<4^{h}$ and $22^{\circ}<\delta<$ $33^{\circ}$, Ichikawa and Fukugita (1992), Watanabe et al. (1992), and Ichikawa and Watanabe (1993) studied the Hubble constant and large-scale peculiar motions 


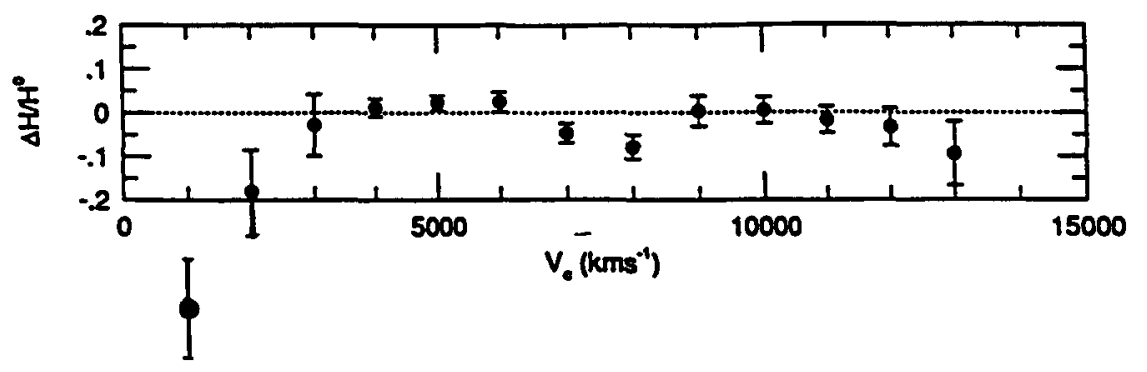

Figure 3. A map of $\delta=\left(H-H_{0}\right) / H_{0}$ in the Pisces-Perseus region (Ichikawa \& Watanabe 1993)

using Tully-Fisher relation in the $B$ band. Taking various biases into account quantitatively, Watanabe et al. (1994) obtained $H_{0}=80 \pm 9_{-22}^{+17} \mathrm{~km} \mathrm{~s}^{-1} \mathrm{Mpc}^{-1}$. The recession of galaxies is described well by a universal Hubble constant for all galaxies up to a distance $c z \sim 13,000 \mathrm{~km} \mathrm{~s}^{-1}$. The prevalent infalling motion of the P-P supercluster towards the Local Group and the Great Attractor was found to be still controversial, after a careful consideration of the biases. A map of the averaged fractional deviations of the local values, $\delta=\left(H-H_{0}\right) / H_{0}$, indicates infall motions toward the supercluster located at about $6,000 \mathrm{~km} \mathrm{~s}^{-1}$ (P-P supercluster) and $11,000 \mathrm{~km} \mathrm{~s}^{-1}$ as well as towards the Local Supercluster (Fig. 3).

Fukugita et al. (1993) carried out a Tully-Fisher analysis in the $B$ band with the complete sample of spiral galaxies in the Virgo cluster of Yasuda et al. (1994). They showed evidence that spiral galaxies in the Virgo cluster are distributed in an elongated filament-like region, which extends from 13 to 30 Mpc. Their result explains the discrepancy in the distance modulus and the dispersion among various authors who carried out Tully-Fisher analyses for the Virgo Cluster.

On the basis of PANBG, Shimasaku and Okamura (1992) studied the velocity field in the Local Supercluster and showed that the standard Great Attractor model proposed by Lynden-Bell et al. (1988) and revised by Faber and Burstein 1988 ) is too simple to delineate the detailed local peculiar velocity field and to discuss whether the Local Anomaly exists.

\section{Conclusion}

At Kiso observatory, we focus on bright nearby galaxies ( $m<17 \mathrm{mag}$ ) for surface photometry, in contrast to surveys for faint galaxies with other large Schmidt telescopes. Efficient and accurate measuring machines and a data reduction system have been developed for the surface photometry of galaxies on the Kiso Schmidt plates. We can determine photometric parameters as accurate as those derived from CCD observations, at least for nearby galaxies. In conclusion, surface photometry of galaxies on Schmidt plates is still useful for 
understanding the local universe, in spite of a lower $\mathrm{S} / \mathrm{N}$ and smaller dynamic range than those of CCD data. The Kiso catalogues provide a new basis for various statistical studies, including the structure of galaxies, cosmic distance scales, and kinematics of the local universe. Moreover, they will serve as a basic reference for future observations of galaxies in the optical with CCD cameras as well as in the near-infrared with a PtSi camera or in other wavelength regions.

\section{References}

Anderson C. M., Slovak M. H. \& Michalski D. E., 1983, PASP, 95, 792

Cornell M. E., Aaronson M., Bothun G. \& Mould J. R., 1987, ApJS, 64, 57

de Vaucouleurs G., 1977, ApJS, 33, 211

de Vaucouleurs G., de Vaucouleurs A., \& Corwin Jr. H. G., 1976, Second Reference Catalogue of Bright Galaxies, (Univ. Texas, Austin) (RC2)

de Vaucouleurs G., de Vaucouleurs A., Corwin Jr. H. G., Buta R. J., Paturel G., \& Fouqué P., 1991, Third Reference Catalogue of Bright Galaxies, (Univ. Texas, Austin) (RC3)

Doi M., 1992, Thesis for Doctor of Science, University of Tokyo, Tokyo

Faber S. M. \& Burstein D., 1988, in Large-Scale Motions in the Universe, V. C. Rubin \& G. V. Coyne, eds, (Princeton Univ. Press, Princeton), p. 116

Fukugita M., Okamura S., Tarusawa K., Rood H. J. \& Williams B. A., 1991 ApJ, 376, 8

Hamabe M. \& Ichikawa S., 1992, Astronomical Data Analysis Software and System I, ASP Conf. Ser. 25, D. M. Worrall et al., eds, p. 325

Ichikawa S., Okamura S., Watanabe M., Hamabe M., Aoki T. \& Kodaira K., 1987, Annals Tokyo Astron. Obs., 21, 285

Ichikawa T. \& Fukugita M., 1992, ApJ, 394, 61

Ichikawa T. \& Watanabe D. M., 1993, in Evolution of the Universie and its Observational Quest, K. Sato, ed., (Universal Academy Press, Tokyo)

Ichikawa T. \& Watanabe D. M., 1994, in preparation

Kodaira K., Okamura S. \& Ichikawa S., 1990, in Photometric Atlas of Northern

Bright Galaxies, (Univ. of Tokyo Press, Tokyo) (PANBG)

Longo G. \& de Vaucouleurs A., 1983, The University of Texas Monograph in Astronomy, No. 3 (Univ. Texas, Austin)

Lauer T. R., 1985, ApJS, 57, 473

Lynden-Bell D., Faber S. M., Burstein D., Davies R. L., Dressler A., Terlevich R. J. \& Wegner G., 1988, ApJ, 326, 19

Pierce M. J. \& Tully R. B., 1988, ApJ, 330, 579 (PT)

Sandage A. \& Tammann G. A., 1981, A Revised Shapley-Ames Catalogue of

Bright Galaxies (Washington: Carnegie Institution of Washington) (RSA)

Shimasaku K., \& Okamura S., 1992, ApJ, 398. 441

Watanabe M., 1983, Annals Tokyo Astron. Obs., 19, 121

Yamagata T., 1986, Annals Tokyo Astron. Obs., 21, 31

Yasuda N., Okamura S. \& Fukugita M., 1994, ApJS, in press 


\section{Discussion}

C. A. Collins: In carrying out Hubble flow studies, it is vital to remove systematic errors in photometry. Do you see any evidence for vignetting on plates from your photographic surface photometry.

T. Ichikawa: As you indicate, systematic errors seriously affect the result of Hubble flow analyses. We, therefore, always take care of such errors. The vignetting of plates can be corrected, dividing pixel values by local background brightness. This method is a kind of flattening with uniform sky background, which is assumed to be constant on a plate.

T. Armandroff: You cited small field size as a disadvantage of CCDs compared to plates. Naturally this is one reason that CCDs have been implemented on Schmidts giving fields up to 1 square degree. Is this field size large enough to answer your concerns for background determination?

T. Ichikawa: The field size of one square degree is large enough for surface photometry of most galaxies except members of the Local Group such as M31 and M33. I feel, however, that a wider field of view is necessary, especially for the study of nearby clusters of galaxies. Larger format array or mosaic array cameras are very useful. 